\title{
Recognition memory for lists of visual stimuli in monkeys and humans
}

\author{
WILLIAM A. ROBERTS and PHILIPP J. KRAEMER \\ University of Western Ontario, London, Ontario N6A 5C2, Canada
}

\begin{abstract}
In Experiment 1, short-term memory for lists of visual stimuli was studied in four squirrel monkeys (Saimiri sciureus). A delayed-matching procedure was used in which a subject was presented with lists containing one, three, or six stimulus patterns, and memory for serial positions was probed by requiring the subject to choose between a list item and a nonlist item. The rate of item presentation was varied, as was the delay between the final item on a list and the retention test. In Experiment 2, the same procedures were used to compare recognition memory in four monkeys and four humans. Although differences in the levels and shapes of the serial-position curves appeared between species, both monkeys and humans showed primacy and recency effects. The presentation time of stimuli had a negligible effect on performance in both monkeys and humans, whereas delay significantly affected human retention but not monkey retention.
\end{abstract}

One of the pervasive phenomena of human memory is the serial-position effect. When people are presented with a list of items, recall typically is best for the final items in the list (recency effect) and the initial items in the list (primacy effect) and worst for items in the middle. The serial-position effect has been observed in serial-anticipation learning of a list of words or nonsense syllables (McCrary \& Hunter, 1953), in memory for a simultaneously presented linear array of stimuli (Jensen, 1962), and in free recall (Murdock, 1962) and recognition (Murdock, 1968) of a single presentation of a list of words.

In several studies of animal memory, subjects have been presented with a list of stimuli, and a recognition memory test was given for one or more members of the list. Studies of this sort with pigeons (MacPhail, 1980; Shimp, 1976; Shimp \& Moffitt, 1974), rats (Roberts \& Smythe, 1979), monkeys (Davis \& Fitts, 1976; Gaffan, 1977), and a dolphin (Thompson \& Herman, 1977) have shown serial-position curves that fall continuously from the end of the list to the beginning. In contrast with the human serial-position curve, these animal studies have shown clear evidence of recency effects but a general absence of primacy effects.

Although these observations suggest the hypothesis that the primacy effect is a uniquely human phenomenon and is not to be found in studies of shortterm recognition memory with animals, a recent set of experiments by Sands and Wright (1980a, 1980b)

Support for this research was provided by Grant A7894 from the Natural Sciences and Engineering Research Council of Canada. Address requests for reprints to W. A. Roberts, Department of Psychology, The University of Western Ontario, London, Ontario N6A 5C2, Canada. discourages this hypothesis. A single rhesus monkey was presented with a list of slide-projected pictures, and memory was tested by presenting a probe item from the list or a picture not presented on the list. The monkey operated a switch in order to respond "same" or "different" to the probe stimulus. In separate experiments with 10 - and 20 -item lists, Sands and Wright discovered serial-position curves containing primacy and recency effects. A human subject tested for comparison scored at a higher level of accuracy than did the monkey, but the shapes of the monkey and human serial-position curves were similar.

Two experiments are reported here, which involved memory for serial lists of visual stimuli in squirrel monkeys. Four monkeys were tested in Experiment 1 using the delayed-matching-to-sample procedure, with lists of one, three, or six stimuli. Memory for serial lists was examined as a function of two variables - rate of item presentation and delay between the final item of a list and the retention test. In the second experiment, both human subjects and monkeys were tested, which allowed us to directly compare human and monkey serial-position curves.

\section{EXPERIMENT 1}

\footnotetext{
Method

Subjects. Four adult squirrel monkeys (Saimiri sciureus) were tested. Three monkeys were females (Dozy, Chick, and Grumpy), and one was male (Stumpy). Prior to the work reported here, Stumpy had served in delayed-matching experiments, but the female monkeys were naive to this procedure.

Apparatus. Testing was carried out in a small primate test chamber, $30.5 \times 21.5 \mathrm{~cm}$ (floor dimensions) $\times 61 \mathrm{~cm}$ (wall height), which was housed within a double-walled, sound attenuating cubicle. The chamber contained a panel with a row of three circular keys; the keys were $2.6 \mathrm{~cm}$ in diameter, were placed $12 \mathrm{~cm}$ above the floor, and were spaced $6 \mathrm{~cm}$ apart, center to center.
} 
Below the keys was a centrally located magazine that dispensed 190-mg Noyes banana and sucrose pellets. A .1-A/28-V houselight was mounted on the panel, $5 \mathrm{~cm}$ above the upper rim of the center key.

Multistimulus projectors mounted behind each key were used to present patterns on the keys. The patterns, shown in Figure 1, consisted of 10 white geometrical designs on black backgrounds. The programming of stimuli, presentation rates, delays, and intertrial intervals was controlled by relays, counters, and timers that were activated by a paper-tape reader. Printing counters recorded choices on each trial.

Procedure. The three female monkeys were trained initially in a darkened chamber to press the two side keys when illuminated with white light for a reinforcer of one banana pellet or one sucrose pellet (the pellet feeder contained approximately a 50:50 mixture of banana and sucrose pellets). In the next stage, a press upon a white center key was required in order to illuminate the side keys. The fixed ratio (FR) on the center key gradually was increased until an FR 10 was reached.

Once monkeys were satisfactorily completing an FR 10 on the center white key and pressing a white side key for reward, simultaneous matching to sample was initiated. On an initial problem, all four monkeys were trained with two of the stimuli shown in Figure 1. On each trial, one stimulus served as the sample and matching stimulus and the other as the nonmatching stimulus, with these stimuli randomly alternating as the matching and nonmatching stimuli across 48 trials of one daily session. A typical trial began with the presentation of a white center key; when the subject had pressed the white center key 10 times, a pattern appeared on this key. Completion of another FR 10 on the center key caused all three keys to be illuminated, the center and one side key with identical sample and matching stimuli and the other side key with the nonmatching stimulus. A press on the matching side key produced $2 \mathrm{sec}$ of illumination of the houselight, the brief sound of a $2,900-\mathrm{Hz}$ tone, and a reward pellet, and a press on the nonmatching key ended the trial without reward. A 20 -sec interval separated trials. Two orders of 48 trials were alternated between daily sessions. Each order contained 24 trials with each stimulus as the sample stimulus, and each stimulus was equally often correct on the left and right side keys.

Once an animal had learned to perform simultaneous matching at $85 \%$ accuracy or better for 5 successive days, it was introduced to a simultaneous matching problem that contained two new stimuli from the set shown in Figure 1. Training continued in this way until a monkey had been trained on five problems and each of the stimuli in the set had been used.

When an animal had completed training on simultaneousmatching problems, delayed matching was begun. An FR 10 again was required on the white center key in order to introduce a pattern; 10 further presses on the center key turned it off and introduced a .5-sec delay, which terminated with illumination of the side keys. Since the center key remained darkened, choice between the matching and nonmatching side keys had to be made from memory. When a monkey had achieved $80 \%-90 \%$ accuracy at the

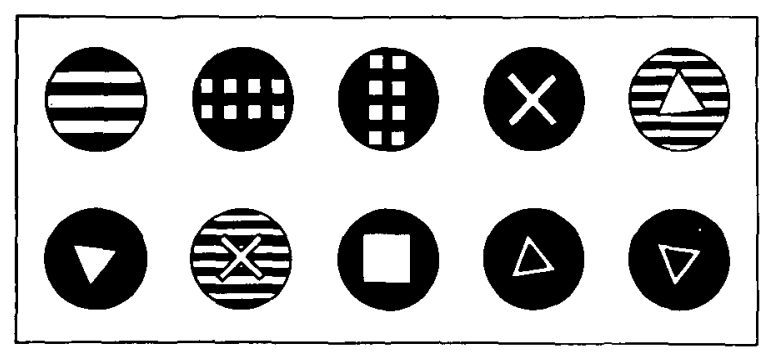

Figure 1. Patterns used as sample and comparison stimuli in Experiments 1 and 2. .5-sec delay, the delay was lengthened to $1 \mathrm{sec}$ and eventually to $2 \mathrm{sec}$. When accurate performance at the 2 -sec delay was reached, the subject was transferred to a new problem. Problems containing all 10 of the stimuli in Figure 1 were tested in this fashion.

When the monkeys were well trained on delayed matching with single sample stimuli, the first experiment was initiated using lists of one, three, or six sample stimuli. Each daily session consisted of 48 trials, with list length $(L L)=1$ tested on 12 trials, $L L=3$ tested on 12 trials, and $L L=6$ tested on 24 trials. On $L L=3$ and $\mathrm{LL}=6$ trials, three or six different sample stimuli were presented sequentially on the center key with the offset of one stimulus followed immediately by the onset of the next. After a delay, retention of one serial position was probed by presenting the stimulus at that position on one side key and a nonmatching stimulus not presented on the list on the other side key. Each serial position within the three- and six-item lists was tested four times within a session. Six different orders of 48 trials were used, with lists within an order constructed by random selection from the pool of 10 stimulus patterns.

Two other variables were examined within the experiment. During preliminary training, an FR-10 requirement had been placed on the center key in order to advance first from white key to pattern and then from pattern to delay. During the experiment, an FR 10 was still required on the white key, but presentation of the list members was controlled by a timer and was independent of the monkeys' presses on the center key. The presentation time (PT) of each stimulus within a list was set at either .5 or $2 \mathrm{sec}$ and was varied factorially with two delays between the final item of a list and the retention test, .5 and $5 \mathrm{sec}$. The four combinations of PT and delay were tested between sessions, and each combination was tested once with each of the six orders of trials within a block of 24 sessions. The experiment was run for four blocks of 24 sessions each.

\section{Results}

Simultaneous matching to sample. The initial problems were learned slowly, but monkeys showed a learning-set effect, since the final problems were learned more rapidly than the early ones. A range of 13 to 44 sessions was required to reach the $85 \%$ criterion on the initial problem, whereas the range for the final problem was 9 to 13 sessions.

Delayed matching to sample. Subjects completed 120 to 135 sessions of training on delayed matching with single sample stimuli at delays of $.5,1$, and $2 \mathrm{sec}$. Accuracy generally ranged from $80 \%-90 \%$ at the .5-sec delay and from $70 \%-85 \%$ at the $1-$ and 2 -sec delays.

Memory for lists. Mean percentage correct responses as a function of serial position for $L L=3,6$, and 1 are shown in Figure 2. Primacy and recency effects can be seen both at $L L=3$ and 6 , and these effects appear under all four combinations of PT and delay, with the exception of a minimal recency effect at $\mathrm{LL}=3$ when $\mathrm{PT}=.5 \mathrm{sec}$ and delay $=5 \mathrm{sec}$. Separate analyses of variance were performed on the data from each list length. With the data from $L L=3$ and 6, the analyses included the factors of serial position, PT, delay, blocks of sessions, and subjects. Serial position was a significant effect at $\mathrm{LL}=3$ $[F(2,6)=18.73, p<.01]$ and $L L=6[F(5,15)=7.91$, $\mathrm{p}<.01]$, but there were no significant effects of PT, delay, or blocks of sessions. Presentation time and delay had no effect on the shapes of the serial-position 


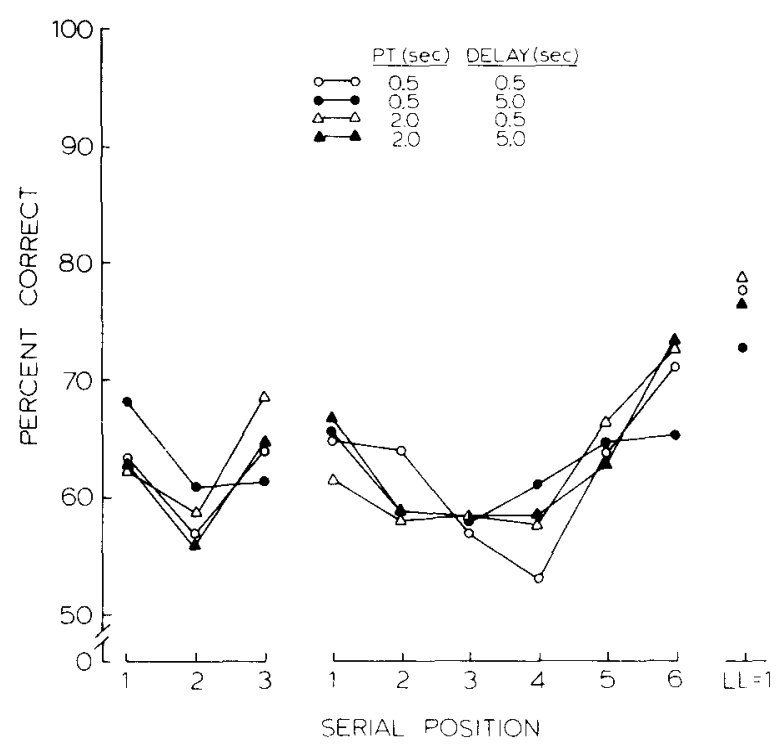

Figure 2. Serial-position curves at list lengths of three, six, and one items for four combinations of presentation time (PT) and delay.

curves, since these variables did not interact significantly with serial position. The data from $L L=1$ were analyzed without the serial position factor, and no significant effects of PT, delay, or blocks of sessions were found.

The data were combined over the PT and delay variables, and post hoc tests were made to compare accuracy at different serial positions on three- and six-item lists. Scheffé (1953) tests were used, and significance was assessed by Rodger's (1975) procedure and tables of critical $F$ values, which limit the number of contrasts that may be declared significant. In the case of $L L=3$, accuracy at Serial Position 1 was significantly greater than that at Serial Position 2 $[F(2,6)=23.16, p<.05]$, and Serial Position 3 was significantly higher than Serial Position $2[F(2,6)=$ $25.45, p<.05$ ]. With the data from $L L=6$, performance at the middle serial positions, 3 and 4 , was compared with the other four positions. Significant differences were found between the middle positions and Serial Positions 1,5 , and $6[\mathrm{Fs}(5,15)=2.87,2.96$, and 10.87 , respectively, ps $<.05]$. Serial Position 2 did not differ significantly from Positions 3 and 4 $[\mathrm{F}(5,15)=.18, \mathrm{p}>.05]$.

The data in Figure 2 suggest that the final item on both the three- and six-item lists may have suffered proactive inhibition (PI), relative to the single item tested at $L L=1$. Proactive inhibition is shown by the fact that each monkey scored higher at $\mathrm{LL}=1$ than at the final serial position in either $L L=3[t(3)=6.35$, $\mathrm{p}<.01]$ or $\mathrm{LL}=6[\mathrm{t}(3)=3.39, \mathrm{p}<.05]$. Apparently, the requirement of processing a list of stimuli detracts from the retention of the final item, relative to a single-item control.
Serial-position curves for individual animals are shown in Figure 3. These curves were obtained by averaging performance over the PT, delay, and blocks of sessions variables. It can be seen that the primacy and recency effects found in the mean curves shown in Figure 2 generally were replicated at the level of each individual subject.

\section{Discussion}

These results reveal serial position curves for monkey memory that contain the primacy and recency effects typically found in many studies of human memory. However, it should be pointed out that the primacy effect often is not found in human recognition memory (Roberts \& Smythe, 1979). Some studies of recognition memory for lists of words or digits have shown a primacy effect (Jahnke \& Erlick, 1968; Murdock, 1962; Shulman, 1970), but others have not (Bjork \& Whitten, 1974; Detterman, 1975; Poltrock \& MacLeod, 1977). Furthermore, experiments that investigate recognition memory for lists of pictures or visual configurations typically have failed to yield primacy effects (Hines, 1975; Nickerson, 1965; Potter \& Levy, 1969; Shaffer \& Shiffrin, 1972; Weaver, 1974; Weaver \& Stanny, 1978). The findings of Sands and Wright (1980a, 1980b) stand as an exception to this conclusion, because their single human subject revealed a primacy effect when tested with the serial probe recognition procedure.

\section{EXPERIMENT 2}

Given a general absence of evidence of a primacy effect in human recognition memory for visual patterns, it would seem premature to conclude that the

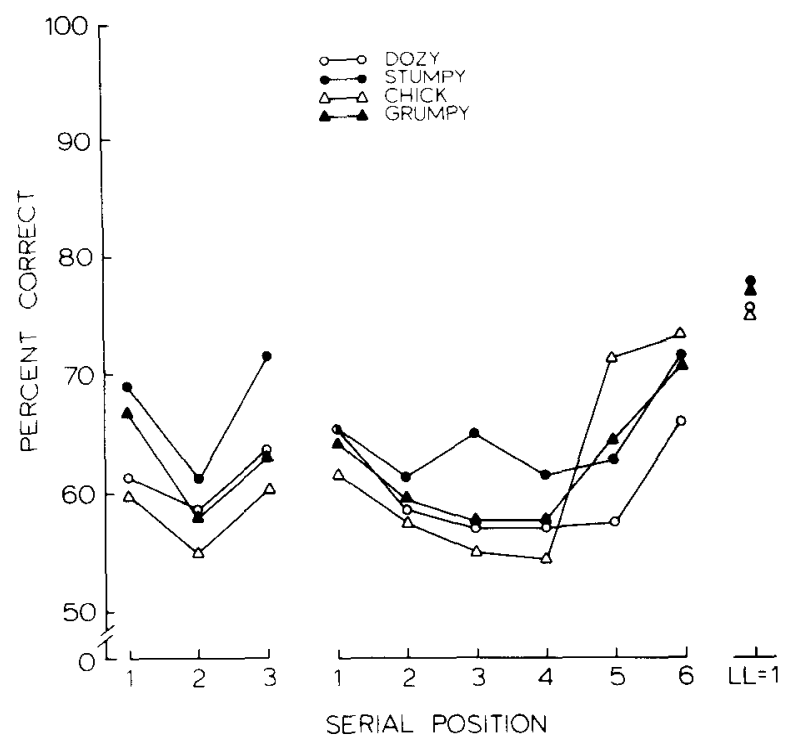

Figure 3. Individual serial-position curves at list lengths of three, six, and one items for four monkeys. 
serial-position curves found with squirrel monkeys in Experiment 1 take the same shape as human curves. The comparability of monkey and human memory in this situation would best be found by testing monkeys and humans under the same conditions. As a result of these considerations, a second experiment was carried out to directly compare human and monkey recognition memory for lists of visual stimuli.

All four monkeys were tested using procedures identical to those used in the first experiment. The PT variable was lowered to values of .25 and $1 \mathrm{sec}$, and delay was set again at values of .5 and $5 \mathrm{sec}$. Four human subjects were tested under conditions highly similar to those used with monkeys. A panel containing three translucent keys was placed on a table, and each subject was tested with the same orders of trials and the same values of PT and delay as were used with monkeys.

\section{Method}

Subjects. The four squirrel monkeys tested in Experiment 1 served as subjects. Four undergraduate students (three women and one man) were tested and paid for their services.

Apparatus. Monkeys were tested with the same equipment used in Experiment 1. For human subjects, a panel of the same dimensions as the one in the operant chamber was placed on a table. This panel was a duplicate of the one used with monkeys, and thus contained three translucent keys in a horizontal row. Projectors mounted behind the keys presented the stimuli seen in Figure 1. When testing human subjects, the control equipment was unplugged from the monkey chamber and plugged into the tabletop panel.

Procedure. Monkeys were tested under the same procedures as were used in the list-testing phase of Experiment 1. The experiment lasted for four blocks of sessions, with each block containing 24 sessions. Six different orders of $\mathbf{4 8}$ trials were used across sessions. Each order contained 12 trials with $L L=1,12$ trials with $L L=3$, and 24 trials with $L L=6$. The presentation rates were faster than in Experiment 1, with the PT variable lowered to values of .25 and $1 \mathrm{sec}$. The delay variable remained at values of .5 and $5 \mathrm{sec}$. The intertrial interval was lowered from $20 \mathrm{sec}$ in Experiment 1 to $10 \mathrm{sec}$ in Experiment 2. The four combinations of PT and delay were tested between sessions, with each combination being tested with each of the six orders of trials within a block of 24 sessions.

Human subjects were tested in a darkened room with the same control equipment and trial orders used with the monkeys. Therefore, the parameters of PT, delay, and intertrial interval used with humans were identical to those used with monkeys. The only difference between the human and monkey tests was that monkeys received a reward pellet for a correct choice and humans did not. However, like the monkeys, humans did receive the brief presentation of a $2,900-\mathrm{Hz}$ tone for a correct choice. The human subjects were instructed to avoid encoding the stimuli with verbal labels and to remember them as visual images. Whereas the monkeys were tested on one session per day, the human subjects were tested irregularly, with subjects sometimes being tested several times in one day and sometimes missing several days between testings. However, like the monkeys, each human subject completed four blocks of 24 sessions.

\section{Results}

Serial-position curves for monkeys and humans are presented in Figure 4. One female monkey, Grumpy, died suddenly after 38 sessions of testing.

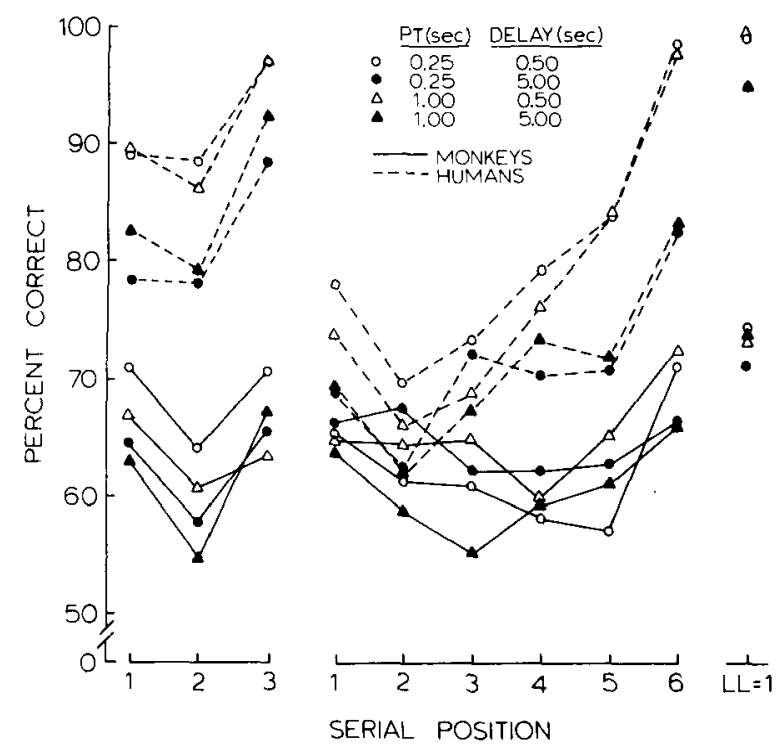

Figure 4. Serial-position curves for monkeys and humans at list lengths of three, six, and one items for four combinations of presentation time (PT) and delay.

The data for these 38 sessions were averaged with the data from the 96 sessions of testing with the other three monkeys in plotting the curves seen in Figure 4. The monkey curves (solid lines) suggest that primacy and recency effects again were revealed with threeand six-item lists. Analyses of variance performed on the monkey data showed significant effects of serial position at both $L L=3[F(2,6)=6.84, p<.05]$ and $L L=6[F(5,15)=5.18, p<.01]$. A post hoc test between serial positions in the three-item list revealed that accuracy was significantly higher at Positions 1 and 3 than at Position $2[F(2,6)=p<.05]$. Within the six-item list, accuracy at Positions 3 and 4 was significantly lower than that at Position $1[F(5,15)=$ 2.32, $p<.05]$ and Position $6[F(5,15)=10.01, p<.05]$ but did not differ significantly from that at Position 2 $[F(5,15)=.87, p>.05]$ or Position $5[F(5,15)=.14$, $p>$.05].

There were no significant main effects of PT or delay at list lengths of one, three, or six items, and these variables failed to interact significantly with serial position on either three- or six-item lists. In the case of the three monkeys that completed all four blocks of sessions, blocks of sessions was a significant effect at $L L=6[F(3,6)=10.54, p<.01]$ but not at $L L=1$ or 3. At $L L=6$, overall accuracy improved from Block $1(61.0 \%)$ to Block $2(64.1 \%)$ and Block $3(65.3 \%)$ but declined somewhat at Block $4(63.0 \%)$.

The PI effect found in the first experiment also was apparent here. Higher retention was found for all four monkeys at $\mathrm{LL}=1$ than at Position 6 on the six-item list $[t(3)=3.26, p<.05]$. This effect failed to reach significance with the three-item list $[\mathrm{t}(3)=1.92$, 
p > .05], because one monkey scored higher at Position 3 than at $\mathrm{LL}=1$.

The human serial-position curves (broken lines) show a higher level of retention than the monkey curves. A primacy effect is barely apparent at $L L=3$ but is strongly evident at $L L=6$, where retention drops from Position 1 to Position 2. A recency effect is seen with both three- and six-item lists, but, unlike the monkey curves at $\mathrm{LL}=6$-which show an extended decline from Position 1 to Positions 3 and 4 and a recency effect at Positions 5 and 6-the human curves demonstrate a continuous rise in accuracy from Position 2 to Position 6. Accuracy varied significantly across serial positions at both $\mathrm{LL}=3[\mathrm{~F}(2,6)$ $=25.39, \mathrm{p}<.01]$ and $L L=6[F(5,15)=14.43$, $\mathrm{p}<.01]$. Post hoc comparisons between serial positions made within the three-item list indicated that accuracy at Position 2 was significantly lower than that at Position $3[\mathrm{~F}(2,6)=40.29, \mathrm{p}<.05]$ but did not differ significantly from Position $1[F(2,6)=$ $1.09, \mathrm{p}>.05]$. Within the six-item list, it was found that performance at Position 2 did not differ significantly from that at Position $3[\mathrm{~F}(5,15)=1.11$, $p>.05]$ but was significantly lower than performance at Positions $1,4,5$, and $6[F s(5,15)=2.18$, $3.76,6.07$, and 25.06, respectively, ps $<.05$ ].

In agreement with the monkey data, humans showed no significant effect of PT at any LL. Unlike the monkeys, however, the 5-sec delay led to significantly lower retention in humans than did the .5 -sec delay at $\mathrm{LL}=3[\mathrm{~F}(1,3)=15.78, \mathrm{p}<.05]$ and $\mathrm{LL}=6$ $[F(1,3)=88.63, p<.01]$ but not at $L L=1[F(1,3)=$ $3.60, p>.05]$. No significant interactions between serial position and PT or delay were found. Blocks of sessions was not a significant factor at $L L=1$ and 3 but was significant at $L L=6[F(3,9)=5.76, p<.05]$. Accuracy dropped across blocks of sessions at $L L=6$; on Blocks 1-4, the percentages of correct responses were $78.9 \%, 74.8 \%, 74.2 \%$, and $72.6 \%$, respectively.

Serial-position curves for individual monkey and human subjects are shown in Figure 5 . It can be seen that the serial-position effects depicted in the mean curves shown in Figure 4 are fairly accurately reflected in the curves for individual subjects.

\section{Discussion}

The major finding of this experiment is that serialposition curves showing both primacy and recency effects were found with both monkey and human subjects. These data confirm the findings of Sands and Wright (1980a, 1980b) with human and monkey subjects and demonstrate that a primacy effect does appear with human subjects using the delayedmatching-to-sample procedure. Nevertheless, some differences between human and monkey performance do appear. Not surprisingly, human subjects were far more accurate than squirrel monkeys. The

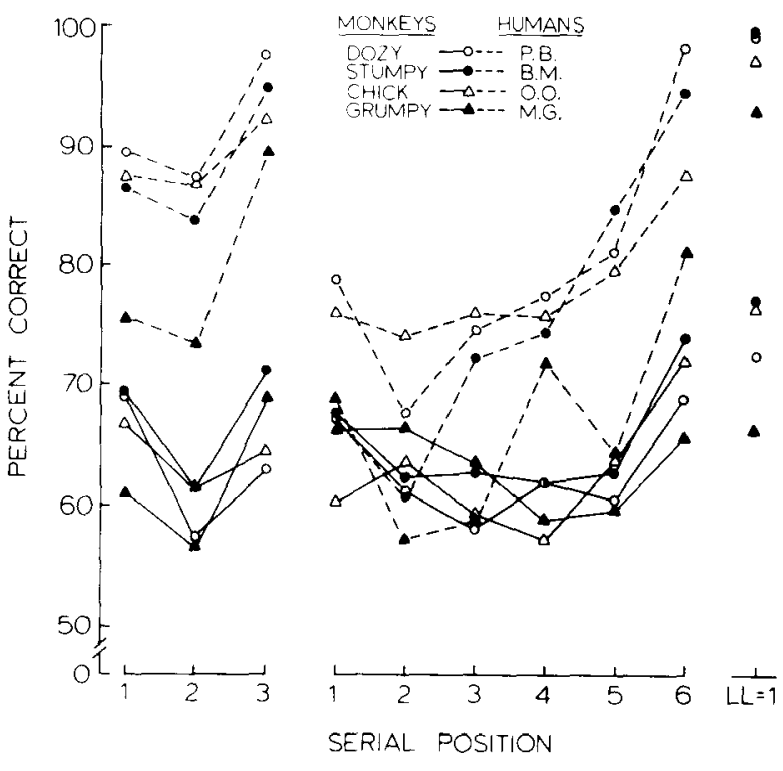

Figure 5. Individual serial-position curves at list lengths of three, six, and one items for four monkeys and four humans.

shapes of the serial-position curves differed also, most notably in the greater primacy effect shown by monkeys than by humans at $L L=3$ and the more marked recency effect shown by humans than by monkeys at $\mathrm{LL}=6$.

Another clear difference between monkeys and humans was found in the effects of the delay variable. The 5-sec delay led to significantly lower accuracy than the .5 -sec delay with human subjects, but delay length had no effect with monkeys. The finding that delay affected human retention is surprising, since previous studies suggest no effect of this variable. Clark (1965) found that recognition of random shapes was unaffected by delays varying between 5 and $20 \mathrm{~min}$, and Hines (1975) reported that recognition memory for random shapes was equally good at no delay and at a 30-sec delay, even when the 30-sec delay was filled with copying and counting distractor tasks.

An important aspect of this experiment is the fact that the visual stimuli used came from the small pool of items shown in Figure 1. Since lists as long as six items were used frequently within a session, there was a great deal of repetition of items between trials. It is well known that such conditions create a situation in which strong PI effects should be found (D'Amato, 1973; Mason \& Wilson, 1974; Worsham, 1975). Also, it is known that PI effects typically increase as a retention interval is made longer (Keppel \& Underwood, 1962). Given these facts, the delay effect found with human subjects may be attributed to PI arising from the frequent use of the same stimuli. The studies of Clark (1965) and Hines (1975) 
used large pools of random shapes, which allowed different stimuli to be presented on each trial.

This analysis of the effect of delay on human subjects leaves a question as to why monkeys were not similarly affected by delay. Previous evidence suggests that highly practiced monkeys would be little affected by delays varying between .5 and $5 \mathrm{sec}$ (D'Amato, 1973). On the other hand, the monkeys in this experiment never reached the high level of performance expected of well-practiced monkeys, even at $L L=1$, and this generally low level of performance may be attributed to PI effects. In fact, Sands and Wright $(1980 \mathrm{a}, 1980 \mathrm{~b})$ attributed the high level of accuracy found with their monkey to the use of a large pool of pictures that minimized PI effects. When Sands and Wright used a small set of pictures, with frequent item repetition between trials, accuracy was reduced markedly. It seems reasonable to suggest that PI effects generally depressed monkeys' retention in this experiment and that a further loss of accuracy at the longer delay may not have appeared as a result of a floor effect. If monkeys' performance could be boosted to the level of human accuracy while still maintaining high PI conditions (which may be impossible), an effect of delay would be expected.

\section{GENERAL DISCUSSION}

The results reported in Experiments 1 and 2 argue strongly that the primacy effect is not limited to human subjects, because both experiments yielded primacy effects in four squirrel monkeys. These findings invite comparison with the results of Sands and Wright (1980a, 1980b). Both laboratories have revealed primacy and recency effects in monkey memory for lists of visual stimuli but under somewhat different conditions. Sands and Wright used one rhesus monkey, a same/different recognition memory procedure, and a large pool of colored slides of natural objects as stimuli, whereas we used four squirrel monkeys, a delayed-matching-to-sample or two-alternative forced-choice procedure, and a small set of black and white geometric patterns as stimuli. The fact that serial-position curves of similar shape were found under these diverse conditions suggests that primacy and recency effects may be general and robust characteristics of primate memory.

Two differences do exist between our findings and those of Sands and Wright. Both Gaffan (1977) and Sands and Wright failed to find a primacy effect with a three-item list. This result prompted Sands and Wright to suggest that a primacy effect would be discovered only with longer lists. Yet, clear and significant primacy and recency effects were found on three-item lists with squirrel monkeys in both Experiments 1 and 2 . The primacy effect appears to be demonstrable at a minimal list length. The reason for this disparity between experiments using a three-item list is not clear at the present time. The second difference between these studies is the achievement of a higher level of accuracy by Sands and Wright's monkey than that achieved by our monkeys.

Although four human subjects were tested in Experiment 2 for purposes of comparison with monkeys, our results on humans and monkeys may be compared with other studies of human recognition memory for lists of visual stimuli. Three differences between our findings and those of other experiments are apparent. The first is that we obtained a significant effect of delay with humans, but other experiments have not (Clark, 1965; Hines, 1975). A second contrast is our failure to find an effect of PT on retention, whereas numerous other studies of human recognition memory for pictures and forms have shown increasing accuracy as a function of the length of time stimuli are exposed (Hines, 1975; Intraub, 1980; Potter, 1976; Potter \& Levy, 1969; Tversky \& Sherman, 1975; Weaver, 1974; Weaver \& Stanny, 1978). A final difference is the demonstration of a primacy effect in our experiments but the absence of a primacy effect in other studies of visual recognition memory (Hines, 1975; Nickerson, 1965; Potter \& Levy, 1969; Shaffer \& Shiffrin, 1972; Weaver, 1974; Weaver \& Stanny, 1978).

A number of the differences found between our results and those of both Sands and Wright and other experiments involving human visual recognition memory may be accounted for by the fact that we used a small pool of stimuli and most other experiments involved the use of large pools of stimuli. It has already been suggested that PI created by the use of a small set of stimuli may be responsible for the lower level of retention shown by our monkeys than by Sands and Wright's monkey and for the significant effect of delay observed with human subjects in Experiment 2. In addition to this difference in PI, set size also may affect speed of encoding stimuli. With frequently repeated stimuli, subjects may become so familiar with the items that encoding may take place in a fraction of a second, with further exposure yielding no additional information. When viewing new stimuli, however, extended visual processing may be necessary in order to get full information into memory. This point also has been made with respect to monkey short-term memory. In agreement with the absence of a PT effect with squirrel monkeys in Experiments 1 and 2, D'Amato and Worsham (1972) found that capuchin monkeys showed equally good delayed matching accuracy at exposure durations varying between .075 and $.45 \mathrm{sec}$. Both D'Amato (1973) and Herzog, Grant, and Roberts (1977) have argued that highly trained monkeys tested with a limited set of stimuli become capable of completely encoding those stimuli at very brief presentation 
times. Given this assumption, it would be expected that PT would have little effect on retention when a highly familiar set of repeated items is used but would become a significant factor when a large set of unfamiliar items is used.

In addition to accounting for differential effects of stimulus exposure time, item familiarity may be used to account for the presence of a primacy effect in our experiments, within the framework of one theoretical model. Atkinson and Shiffrin (1980) and Rundus (1971) have postulated that the initial members of a list of verbal items are rehearsed during both their own presentation and that of subsequent items and that extra rehearsal of initial items increases their transfer from short-term store to long-term store. In a list containing unfamiliar visual items, each item might be processed for the entire period it is presented and differential rehearsal would not occur. With familiar and rapidly encoded stimuli, such as words or well-known pictures or patterns, rehearsal of initial items could take place during presentation of subsequent items. At first, this hypothesis may seem to be out of line with the fact that Sands and Wright found a primacy effect with monkey and human subjects using a large pool of 211 slides. However, these items were used repeatedly across testing sessions, and subjects had ample opportunity to become familiar with them. If item familiarity is the critical factor responsible for the primary effect, we would expect that monkeys tested with continually novel stimuli would not show a primacy effect.

Of course, the use of the two-store model to account for the primacy effects seen in Experiments 1 and 2 involves the assumption that memories of visual stimuli are rehearsed by monkeys, as well as humans. Other processes have been suggested to account for serial-position effects, such as perceptual organization (Asch, Hay, \& Diamond, 1960) and superior retrieval cues for initial and final items (Tulving, 1968; Wagner \& Pfautz, 1978). Although the experiments presented here are not sufficiently analytical to allow us to begin to theorize in depth about the basis for primacy and recency effects in the monkey, these results appear to broaden the range of species toward which theoretical efforts will have to be directed. The notion that a primacy effect is a general characteristic of primate memory may have important implications for the form that a theory or model might take. For example, any theory based on verbal processing that offered a general account of the serial-position curve would encounter difficulties with the monkey findings. Some theorists may wish to entertain the possibility that several processes can yield primacy effects and that these processes may operate differentially depending upon the experimental situation, stimulus materials, and species tested.

While the results reported here and by Sands and
Wright suggest that the primacy effect may be characteristic of primate memory in general, the question now arises as to whether this effect will be found with nonprimate species. Recency effects have been reported with pigeons (MacPhail, 1980; Shimp, 1976; Shimp \& Moffitt, 1974), rats (Roberts \& Smythe, 1979), rabbits (Whitlow, 1975), and a dolphin (Thompson \& Herman, 1977), but none of these studies found any evidence of primacy effects. Wagner and Pfautz (1978) have reported a bowed serial-position curve for retention of the habituation of a vasoconstrictive response in rabbits, but this experiment involved repeated presentation of a list prior to testing. It seems to remain an open question whether shortterm memory for the single presentation of a list of stimuli will yield a primacy effect in nonprimate species.

\section{REFERENCES}

Asch, S. E., HaY, J., \& Diamond, R. M. Perceptual organization in serial rote-learning. American Journal of Psychology, 1960, 73, 177-198.

Atkinson, R. C., \& Shiffrin, R. M. Human memory: A proposed system and its control processes. In K. W. Spence \& J. T. Spence (Eds.), The psychology of learning and motivation (Vol. 2). New York: Academic Press, 1968.

BjoRK, R. A., \& Whitten, W. B. Recency-sensitive retrieval processes in long-term free recall. Cognitive Psychology, 1974, 6, 173-189.

Clark, H. J. Recognition memory for random shapes as a function of complexity, association value, and delay. Journal of Experimental Psychology, 1965, 69, 590-595.

D'Aмато, M. R. Delayed matching and short-term memory in monkeys. In G. H. Bower (Ed.), The psychology of learning and motivation (Vol. 7). New York: Academic Press, 1973.

D'Aмato, M. R., \& Worsham, R. W. Delayed matching in the capuchin monkey with brief sample durations. Learning and Motivation, 1972, 3, 304-312.

Davis, R. T., \& Fitrs, S. S. Memory and coding processes in discrimination learning. In D. L. Medin, W. A. Roberts, \& R. T. Davis (Eds.), Processes of animal memory. Hillsdale, N.J: Erlbaum, 1976.

DetTerman, D. K. The Von Restorff effect and induced amnesia: Production by manipulation of sound intensity. Journal of Experimental Psychology: Human Learning and Memory, 1975, 1, 614-628

GAFFAN, D. Recognition memory after short retention intervals in fornix-transected monkeys. Quarterly Journal of Experimental Psychology, 1977, 29, 577-588.

Herzog, H. L., Grant, D. S., \& Roberts, W. A. Effects of sample duration and spaced repetition upon delayed matchingto-sample in monkeys (Macaca arctoides and Saimiri sciureus). Animal Learning \& Behavior, 1977, 5, 347-354.

HINES, D. Immediate and delayed recognition of sequentially presented random shapes. Journal of Experimental Psychology: Human Learning and Memory, 1975, 1, 634-639.

Intraus, H. Presentation rate and the representation of briefly glimpsed pictures in memory. Journal of Experimental Psychology: Human Learning and Memory, 1980, 6, 1-12.

JAHNKE, J. C., \& ERLICK, D. E. Delayed recognition and the serial organization of short-term memory. Journal of Experimental Psychology, 1968, 77, 641-647.

Jensen, A. R. Temporal and spatial effects of serial position. American Journal of Psychology, 1962, 75, 390-400. 
Keppel, G., \& Underwood, B. J. Proactive inhibition in shortterm retention of single items. Journal of Verbal Learning and Verbal Behavior, 1962, 1, 153-161.

MacPhaIl, E. M. Short-term visual recognition memory in pigeons. Quarterly Journal of Experimental Psychológy, 1980, 32, $521-538$.

Mason, M., \& Wilson, M. Temporal differentiation and recognition memory for visual stimuli in rhesus monkeys. Journal of Experimental Psychology, 1974, 103, 383-390.

MoCrary, J. W., \& Hunter, W. S. Serial position curves in verbal learning. Science, 1953, 117, 131-134.

MuRdock, B. B., JR. The serial position effect in free recall. Journal of Experimental Psychology, 1962, 64, 482-488.

Murdock, B. B., JR. Modality effects in short-term memory: Storage or retrieval? Journal of Experimental Psychology, 1968, 77, 79-86.

Nickerson, R. S. Short-term memory for complex meaningful visual configurations: A demonstration of capacity. Canadian Journal of Psychology, 1965, 19, 155-160.

Poltrock, S. E., \& MAcLeod, C. M. Primacy and recency in the continuous distractor paradigm. Journal of Experimental Psychology: Human Learning and Memory, 1977, 3, 560-571.

PotTe R, M. C. Short-term conceptual memory for pictures. Journal of Experimental Psychology: Human Learning and Memory, 1976, 2, 509-522.

Potten, M. C., \& Levy, E. I. Recognition memory for a rapid sequence of pictures. Journal of Experimental Psychology, $1969,81,10-15$.

Roberts, W. A., \& Smythe, W. E. Memory for lists of spatial events in the rat. Learning and Motivation, 1979, 10, 313-336.

Rodoer, R. S. The number of non-zero, post hoc contrasts from ANOVA and error-rate. I. British Journal of Mathematical and Statistical Psychology, 1975, 28, 71-78.

Rundus, D. Analysis of rehearsal processes in free recall. Journal of Experimental Psychology, 1971, 89, 63-77.

SAnds, S. F., \& Wriart, A. A. Primate memory: Retention of serial list items by a rhesus monkey. Science, 1980, 209, 938939. (a)

Sand8, S. F., \& Wright, A. A. Serial probe recognition performance by a rhesus monkey and a human with 10 and 20 -item lists. Journal of Experimental Psychology: Animal Behavior Processes, 1980, 6, 386-396. (b)
Scherré, H. A method for judging all contrasts in the analysis of variance. Biometrika, 1953, $40,87-104$.

Shatren, W. O., \& Shiffrin, R. M. Rehearsal and storage of visual information. Journal of Experimental Psychology, 1972, 92, 292-296.

ShIMP, C. P. Short-term memory in the pigeon: Relative recency. Journal of the Experimental Analysis of Behavior, 1976, 25, S5-61.

Shimp, C. P., \& MoffitT, M. Short-term memory in the pigeon: Stimulus-response associations. Journal of the Experimental Analysis of Behavior, 1974, 22, 507-512.

ShuLwan, H. G. Encoding and retention of semantic and phonemic information in short-term memory. Journal of Verbal Learning and Verbal Behavior, 1970, 9, 499-508.

ThoM Pson, R. K. R., \& HeRMAN, L. M. Memory for lists of sounds by the bottle-nosed dolphin: Convergence of memory processes with humans? Science, 1977, 195, 501-503.

Tulving, E. Theoretical issues in free recall. In T. R. Dixon \& D. L. Horton (Eds.), Verbal behavior and general behavior theory. Englewood Cliffs, N.J: Prentice-Hall, 1968.

Tversxy, B., \& Sherman, T. Picture memory improves with longer on time and off time. Journal of Experimental Psychology: Human Learning and Memory, 1975, 104, 114-118.

Wagner, A. R., \& Prautz, P. L. A bowed serial-position function in habituation of sequential stimuli. Animal Learning \& Behavior, 1978, 6, 395-400.

WeAver, G. E. Effects of poststimulus study time on recognition of pictures. Journal of Experimental Psychology, 1974, 103, $799-801$.

Weaver, G. E., \& Stanny, C. J. Short-term retention of pictorial stimuli as assessed by a probe recognition technique. Journal of Experimental Psychology: Human Learning and Memory, 1978, 4, 55-65.

WhirLow, J. W. Short-term memory in habituation and dishabituation. Journal of Experimental Psychology: Animal Behavior Processes, 1975, 1, 189-206.

Worsham, R. W. Temporal discrimination factors in the delayed matching-to-sample task in monkeys. Animal Learning \& $\mathrm{Be}$ havior, 1975, 3, 93-97.

(Manuscript received January 6, 1981; revision accepted for publication June 18,1981 .) 